\title{
Rates of microfilarial production by Onchocerca volvulus are not cumulatively reduced by multiple ivermectin treatments
}

\author{
CHRISTIAN BOTTOMLEY ${ }^{1} \uparrow$, VALERIE ISHAM ${ }^{2}$, RICHARD C. COLLINS ${ }^{3}$ \\ and MARIA-GLORIA BASÁÑEZ ${ }^{4 *}$ \\ ${ }^{1}$ Department of Primary Care \& Population Sciences, Royal Free Hospital, Rowland Hill Street, London NW3 $2 P F$ \\ ${ }^{2}$ Department of Statistical Science, University College London, Gower Street, London WC1E 6BT \\ ${ }^{3}$ P.O. Box 715, Sonoita, AZ 85637, USA \\ ${ }^{4}$ Department of Infectious Disease Epidemiology, Imperial College London, St. Mary's Campus, Norfolk Place, \\ London W2 $1 P G$
}

(Received 30 May 2008; revised 13 August 2008; accepted 13 August 2008; first published online 3 October 2008)

\begin{abstract}
SUMMARY
Regular distribution of ivermectin reduces onchocerciasis transmission and morbidity by killing, within humans, the microfilarial stage of the parasite (microfilaricidal effect). In addition, ivermectin exerts a so-called embryostatic effect by which microfilarial production by the adult female worm becomes suppressed during a number of weeks after treatment. To assess the overall effect of ivermectin on onchocerciasis transmission and evaluate the likelihood of local elimination of the infection it is important to estimate the magnitude of the anti-fertility effect over the course of a treatment programme. Estimates of the effect of repeated drug treatments on the production of microfilariae by adult Onchocerca volvulus were obtained by developing a model that was fitted to data collected from three hyperendemic communities in Guatemala, where eligible residents received ivermectin twice per year for two and a half years. The data consist of microfilarial load measurements in the skin, collected just before each six-monthly treatment during the programme. The model that is developed describes the dynamics of an individual host's expected microfilarial load over the 30-month study period. We adopt a Bayesian approach and use Markov chain Monte Carlo (McMC) techniques to fit the model to the data. Combining estimates from the three villages, average microfilarial production in the first six months post-treatment was reduced by $\sim 64 \%$ of its pre-treatment level, regardless of values chosen for the pre-ivermectin fertility rate within plausible ranges. Increased adult worm death rate after treatment (to mimic removal of macrofilariae via nodulectomy during the programme) resulted in a smaller estimated magnitude of the embryostatic effect (rate of microfilarial production was reduced by $\sim 58 \%$ of pre-ivermectin value). After subsequent treatments, the rate of microfilarial production appeared to be similarly decreased. The data and analyses therefore do not support the hypothesis of a cumulative effect of multiple ivermectin treatments on microfilarial production by female worms.
\end{abstract}

Key words: Onchocerca volvulus, microfilariae, multiple ivermectin treatments, Bayesian hierarchical model, Guatemala.

\section{INTRODUCTION}

Mass administration with the microfilaricidal drug ivermectin (Mectizan) has been a cornerstone for onchocerciasis control for the last two decades, since it was licensed for human use in the late 1980s and donated for as long as necessary to eliminate the burden of onchocerciasis by Merck \& Co. (Meredith and Dull, 1998). In the Onchocerciasis Control Programme in West Africa (OCP), ivermectin was initially used in conjunction with vector control in some areas and as a sole control measure in others

* Corresponding author: Department of Infectious Disease Epidemiology, Imperial College London, St. Mary's Campus, Norfolk Place, London W2 1PG. Tel: +44 (0)207594 3295. E-mail: m.basanez@imperial.ac.uk $\dagger$ Current address: Infectious Disease Epidemiology Unit, London School of Hygiene and Tropical Medicine, Keppel Street, London, WC1E 7HT
(Molyneux and Davies, 1997). In the African Programme for Onchocerciasis Control (APOC), operational in those countries not covered by the OCP, and in the Onchocerciasis Elimination Program for the Americas (OEPA), control relies mainly on the long-term and regular treatment of endemic populations with ivermectin (annually in APOC and biannually in OEPA; Richards et al. (2001)), with a few focal vector elimination projects in APOC.

Regular treatment with ivermectin reduces the microfilarial load in the skin of infected individuals. This leads to substantial reductions in morbidity, transmission of the parasite to vectors, and eventually of the force of infection (see Basáñez et al. (2006) for a recent review). Within 14 days of treatment, microfilarial loads are typically reduced to $1 \%$ of pretreatment levels (Diallo et al. 1986).

In addition to a microfilaricidal effect, treatment with ivermectin exerts a so-called embryostatic 
effect, by which the production of live microfilariae by adult Onchocerca volvulus female worms is inhibited for several weeks and occurs at a reduced rate during subsequent months (Duke, Zea-Flores and Muñoz, 1991). Basáñez et al. (2008) have conducted a systematic review and mathematical modelling of meta-analysed data both for the microfilaricidal and embryostatic effects of single-dose ivermectin, and their results agreed with those of Plaisier et al. (1995) who found that about 10-12 months after treatment microfilarial production would have recovered only to $\sim 65-70 \%$ of its initial value. However, the question still remains as to whether or not this effect is cumulative, i.e. whether subsequent ivermectin treatments lead to further reductions in microfilarial production (Kläger et al. 1993). The assumption that rates of microfilarial production are progressively reduced with each treatment round has been central to the predictions, derived from the microsimulation ONCHOSIM model that onchocerciasis can be eliminated from African settings with the sole use of ivermectin as a control measure (Winnen et al. 2002). Since OEPA is an elimination and not only a morbidity reduction programme, and since treatment is administered twice per year instead of annually in Latin American settings, we set out to investigate the anti-fertility effect of multiple doses of ivermectin administered during a field trial in Central America (Collins et al. 1992). In this trial, the residents of five hyperendemic communities (coffee fincas) in the central onchocerciasis focus of Guatemala received ivermectin every 6 months for 30 months for a total of five treatment rounds.

To estimate rates of microfilarial production we develop a model for the dynamics of expected microfilarial load by way of a model for worm burden during the 30-month study period. The model incorporates both the death of adult worms as well as parasite transmission. To fit the model to data, we adopt a hierarchical Bayesian approach using Markov chain Monte Carlo (McMC) to simulate from the posterior distribution of parameters. This work follows on from that of Plaisier et al. (1995) who examined rates of microfilarial production by fitting a mathematical model to data obtained during a field trial conducted in the Asubende region of Ghana, where five ivermectin treatment rounds were administered annually during a 60-month study period (Alley et al. 1994).

MODELS AND METHODS

\section{Study area and parasitological procedures}

We use data on microfilarial loads recorded by Collins et al. (1992) from three coffee fincas (namely, Los Tarrales, Santa Emilia and Los Andes) located in the vicinity of Lake Atitlán, in the central onchocerciasis focus of Guatemala. The original study comprised five hyperendemic villages (microfilarial prevalence $\geqslant 60 \%$ ), including also El Vesubio and
Santa Isabel. In our analysis, we excluded data from the latter two communities because of the small population size in El Vesubio, and the low compliance to skin-snipping and treatment in Santa Isabel. Also, El Vesubio received placebo during the first three treatments, and ivermectin only during the last two rounds (Collins et al. 1992).

Five ivermectin treatment rounds were administered to the eligible population of these communities at six-month intervals between May 1988 and May 1990. Two skin-snips were taken to measure microfilarial burden immediately prior to each treatment round: one from the left shoulder, and the other from the left iliac crest. A $2.0 \mathrm{~mm}$ corneoscleral punch was used to obtain the samples, and they were subsequently incubated for $24 \mathrm{~h}$ in physiological saline. Skin-snip processing and weighing, and microfilarial counts were done following the methods of Brandling-Bennett et al. (1981). On the occasion of each parasitological examination, subcutaneous nodules (onchocercomata) were palpated and recorded on a body chart for each individual. Since at the time of the original study the only accepted form of treatment was excision of detectable nodules, nodulectomy was offered to the participants on a voluntary basis and undertaken by nodulectomy brigades, sometimes weeks or months after skin-snipping and treatment. In fact, after the second treatment round, skin-snipping and ivermectin were disassociated from nodulectomy in order to increase compliance (Collins et al. 1992). We do have nodulectomy data on the subjects that volunteered for the time period of the study but the excised nodules were not analysed for their worm content. Therefore, we lack individual host data on the precise number of adult worms actually removed. However, since it is biologically reasonable to suppose that removal of nodules would cause some degree of excess adult worm mortality, its possible effects were accounted for by assessing the sensitivity of model outputs to variation in the per macrofilaria (adult worm) death rate (see sensitivity analysis section).

On average, treatment coverage of the census population was $67 \cdot 1 \%$ in Los Tarrales, $61 \cdot 1 \%$ in Santa Emilia, and $75.5 \%$ in Los Andes. In our analysis we used data only from individuals who received treatment at all five timepoints $(\mathrm{N}=267$ in Los Tarrales, $\mathrm{N}=119$ in Santa Emilia, and $\mathrm{N}=124$ in Los Andes). Further details of the epidemiological, parasitological, and entomological characteristics of these fincas, and of the methods employed during the surveys can be found in Collins et al. (1992) and Cupp et al. (1992).

\section{A deterministic model of mean worm burden}

The following deterministic model describes the evolution of mean worm burden in a population of hosts (see Fig. 1). 


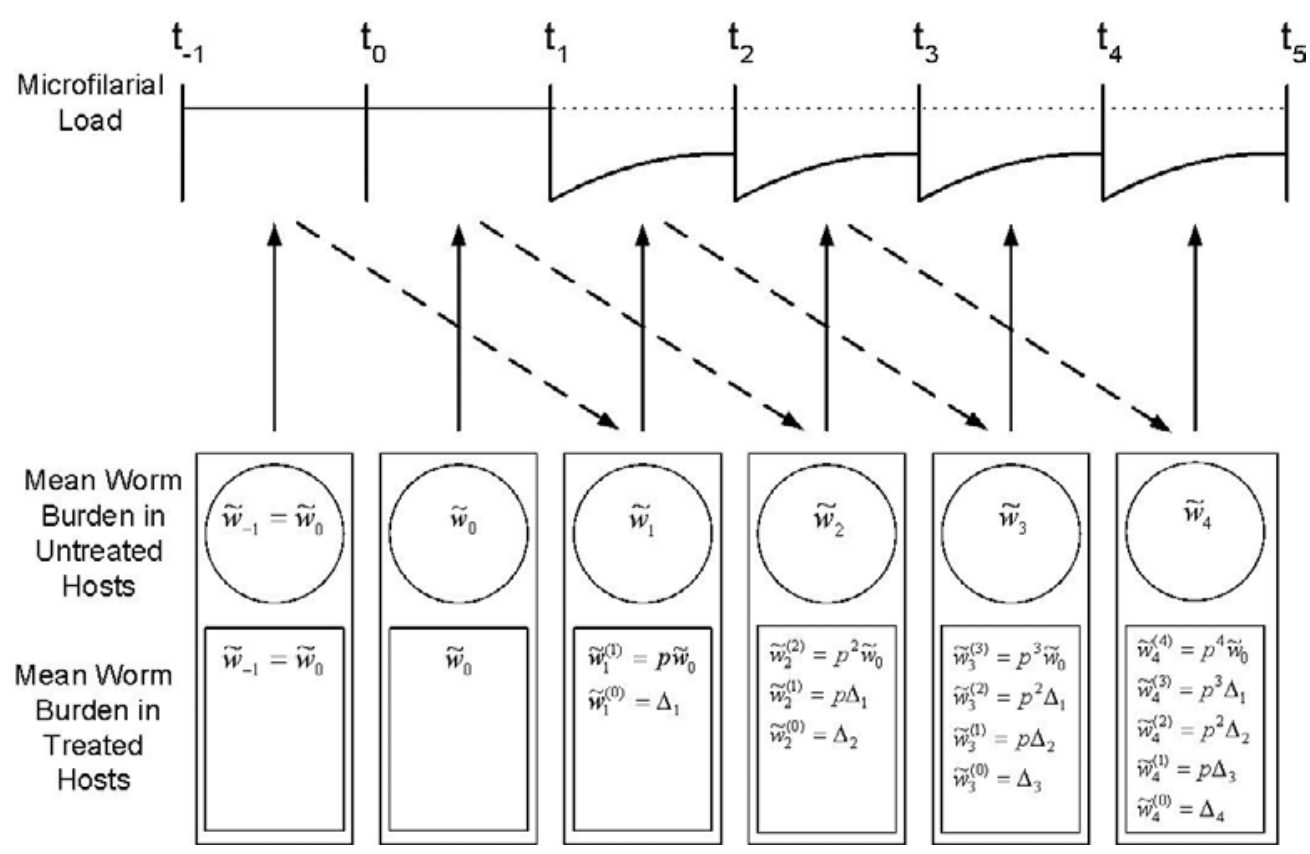

Fig. 1. A model for the evolution of mean worm burden in the host population. The rate of microfilarial production by an adult worm depends on the number of treatments experienced. The mean number of worms having received $n$ treatments is denoted by $\tilde{w}_{j}^{(n)}$ and the average worm burden in untreated hosts is $\tilde{w}_{j}$. Treatments occur at six-month intervals with the first treatment at $t_{1}$. The solid and dotted lines represent mean microfilarial loads in treated and untreated hosts respectively. The solid arrows represent the effect of mean worm burden in treated and untreated hosts on mean microfilarial load, and the dashed arrows relate microfilarial load to infection and the establishment of adult worms after a 12 month maturation period. Thus the average number, $\Delta_{j}$, of adult worms newly acquired by a host during the interval $\left(t_{j}, t_{j+1}\right)$ depends on mean microfilarial load in the host population 12 months previously in the period $\left(t_{j-2}, t_{j-1}\right)$.

We model worm burden in discrete time, with time steps every six months. The five treatment times occur at times $t_{1}, \ldots, t_{5}$. Between treatments a proportion, $p$, of the worms die for reasons unrelated to treatment. In general we suppose that for $t$ in the interval $\left(t_{j}, t_{j+1}\right)$ there are on average $\tilde{w}_{j}{ }^{(n)}(t)$ adult worms per host that have survived $(n)$ rounds of treatment $(n=0, \ldots, j)$. Here $n$ tracks both the number of treatment rounds and the worm's age in multiples of 6 months. Since treatment occasions correspond to times when microfilaridermia was assessed, the model described below enables observed microfilarial load to be linked to unobserved worm burden, appropriately lagged. Prior to treatment $\left(t<t_{1}\right)$ we set mean worm burden to be constant so that $\tilde{w}_{j}^{(\mathbf{0})}(t)=\tilde{w}_{0}$ when $j<1$ (this assumption is satisfied if the distribution of worm burden is at equilibrium before treatment). After the start of the treatment programme $(j \geqslant 1)$ mean worm burden is modelled using the following difference equation (time-dependence has been omitted to simplify the notation)

$$
\begin{aligned}
& \tilde{w}_{j}^{\left({ }^{(0)}\right.}=\Delta_{j} \\
& \tilde{w}_{j}^{(n)}=p \tilde{w}_{j-1}^{(n-1)} \quad(n=1, \ldots, j)
\end{aligned}
$$

where $\Delta_{j}$ is the average number of adult worms newly acquired per host in $\left(t_{j}, t_{j+1}\right)$ that survive to the end of this period, and $p$ is the fraction of established worms that survive the interval between treatments. The parameter $\Delta_{j}$ depends on microfilarial loads within both the treated and untreated host populations, since microfilariae are transmitted to the simuliid vectors where they develop into stages infective to humans.

Among treated $(+)$ hosts average microfilarial load per mg of skin, $\tilde{m}_{j}^{+}(t)$, is set to zero at treatment time $t_{j}$, i.e. $100 \%$ microfilaricidal efficacy is assumed. Subsequently, for $t$ in $\left(t_{j}, t_{j+1}\right)$ the change in average microfilarial load is

$\frac{d \tilde{\boldsymbol{m}}_{j}^{+}}{d t}=\frac{1}{2} \sum_{n=\mathbf{0}}^{j} \phi^{(n)} \tilde{\mathfrak{w}}_{j}^{(n)}-\mu_{m} \tilde{\boldsymbol{m}}_{j}^{+}$

where $\tilde{w}_{j}^{(n)} / 2$ is the average number of female worms per host (assuming a 1:1 sex ratio according to Schulz-Key and Karam (1986)); $\phi^{(n)}$ is the rate of microfilarial production per mated female worm after $n$ rounds of treatment, and $\mu_{m}$ is the per capita death rate of microfilariae. We ignore the impact of human mortality on mean microfilarial load as the study period is short relative to human lifeexpectancy.

The solution to eqn 2 is,

$\tilde{\boldsymbol{m}}_{j}^{+}(t)=\frac{1-e^{-\mu_{m}\left(t-t_{j}\right)}}{2 \mu_{m}} \sum_{n=\mathbf{0}}^{j} \phi^{(n)} \tilde{w}_{j}^{(n)}$. 
For untreated (-) hosts we assume that the mean microfilarial load, $\tilde{m}_{j}^{-}(t)$ has reached an equilibrium so that for this group,

$\tilde{m}_{j}^{-}(t)=\phi^{(0)} \tilde{w}_{j} / 2 \mu_{m}$

where $\tilde{w}_{j}$ is the average worm burden among untreated hosts. Since the dynamics of mean worm burden is the same in the treated and untreated host populations (assuming no macrofilaricidal effect of ivermectin), we let $\tilde{w}_{j}=\sum_{n=0}^{j} \tilde{w}_{j}^{(n)}$.

For the host population as a whole (both treated and untreated hosts) the average microfilarial load during $\left(t_{j}, t_{j+1}\right)$ is

$\tilde{m}_{j}(t)=f_{j} \tilde{m}_{j}^{+}(t)+\left(1-f_{j}\right) \tilde{m}_{j}^{-}(t)$

where $f_{j}$ is the fraction of hosts treated at the $j$ th treatment. Prior to treatment $(j<1)$ by definition. $f_{j}=0$. For $j \geqslant 1$ we use treatment coverages of the total, census populations, as reported in Collins et al. (1992) for each treatment round and within each community.

Transmission of the parasite between hosts occurs via the bites of blackfly (Diptera: Simuliidae) vectors. In Guatemala, Simulium ochraceum Walker s.l. is the main blackfly species responsible for transmission (Collins, 1979). We chose not to model this process explicitly for the purposes of this paper. Instead, we note that the life-expectancy of the vectors and of the larvae within them are short relative to that of microfilariae and adult worms within humans, and assume that transmission between hosts occurs instantaneously. Such an assumption has also been made in other models of filariases transmission (Duerr, Dietz and Eichner, 2005).

Following infection of humans, the L3 infective larvae mature to become adult worms; this is incorporated into the model through a time delay. In particular, the rate at which worms are newly acquired, encapsulated by the parameter $\Delta_{j}$, is assumed to depend on average microfilarial loads in the population of hosts 12 months earlier (Duke, 1980) during the period $\left(t_{j-2}, t_{j-1}\right)$. (The sensitivity of model outcomes to varying incubation periods of 6,12 , and 18 months was low when explored for an earlier version of the model.) Thus we use the following expression for $\Delta_{j}$

$\Delta_{j}=\int_{t_{j-2}}^{t_{j-1}} q \tilde{m}_{j-2}(t) e^{-\mu_{v v}\left(t_{j-1}-t\right)} d t$

where $q$ is the product of the per microfilaria rate of transmission from one human to another human (via the vector) and the probability that an inoculated L3 larva survives the process of maturation within the human host. Note that the rate of human to human transmission itself depends proportionally on the following: (1) the ratio of vectors to humans; (2) the square of the biting rate per fly on humans;
(3) the probability of establishment of microfilariae within vectors and of L3 larvae within humans, and (4) the life-expectancy of parasites within surviving vectors. In reality, $q$ is likely to be density-dependent (Collins et al. 1977; Basáñez et al. 1995, 2002, 2007; Duerr et al. 2003), but we ignore this complexity in the modelling approach presented here and expound on its implications in the Discussion. The term $e^{-\mu_{v v}\left(t_{j-1}-t\right)}$ represents the probability that a worm newly acquired at time $t$ survives to $t_{j-1}\left(\mu_{w}\right.$ is the per capita death rate of worms).

Combining eqns $3-6$, we obtain

$$
\begin{aligned}
\Delta_{j}= & \frac{q\left(1-f_{j-2}\right)}{2 \mu_{m} \mu_{w}}\left(1-e^{-\mu_{w} s}\right) \phi^{(0)} \tilde{w}_{j-2} \\
& +\frac{q f_{j-2}}{2 \mu_{m}}\left(\frac{\left(1-e^{-\mu_{w s} s}\right)}{\mu_{w}}-\frac{\left(e^{-\mu_{w} s}-e^{-\mu_{m} s}\right)}{\mu_{m}-\mu_{w}}\right) \\
& \times \sum_{n=\mathbf{0}}^{j-2} \phi^{(n)} \tilde{w}_{j-2}^{(n)}
\end{aligned}
$$

where $s=\left(t_{j-1}-t_{j-2}\right)$.

For $j<3$ eqn 7 simplifies to become

$\Delta_{j}=\frac{q\left(1-e^{-\mu_{w} s}\right) \phi^{(0)} \tilde{w}_{0}}{2 \mu_{m} \mu_{w}}$

When $j<3$, we must have that $\tilde{w}_{0}=\Delta_{j}+p \tilde{w}_{0}$ since we are assuming that mean worm burden is constant prior to treatment. It therefore follows that $\Delta_{j}=\left(1-e^{-\mu_{w} s}\right) \tilde{w}_{0}$ and $q=2 \mu_{m} \mu_{w} / \phi^{(0)}$ for $j<1$.

For $j \geqslant 1$, the parameter $q$ is unlikely to remain constant since it will almost certainly depend on the level of worm burden and the density of various parasite life-stages. However, we will assume that changes in worm burdens are sufficiently small during the 30 -month study period so that we may set $q=2 \mu_{m} \mu_{w} / \phi^{(0)}$ for $j=1, \ldots, 5$.

\section{Expected microfilarial load within an individual host}

The model of the previous section can be extended to describe the expected dynamics of worm burden and microfilarial load within individual hosts. (In the context of the analysis presented in this paper the term 'expectation' or 'expected value' refers to the mean or average value of the random variable in question.) In the following description, the dynamics of mean worm burden and microfilarial load within the host mimic the dynamics of mean worm burden across the population of hosts, except that we allow each host to have a different initial worm burden $\left(w_{0}\right)$. By extending the model to the individual, it can be fitted to individual-level data on microfilarial loads.

Let $w_{j}^{(n)}(t)$ denote the expected number of worms within a host at time $t$ in the interval $\left(t_{j}, t_{j+1}\right)$ that have survived $(n)$ rounds of treatment and set 
$w_{0}^{(0)}=w_{0}$, prior to treatment $\left(t<t_{1}\right)$. Following treatment $(j \geqslant 1)$ we have

$w_{j}^{(0)}=\Delta_{j}$

$w_{j}^{(n)}=p w_{j-1}^{(n-1)} \quad(n=1, \ldots, j)$

where $p$ is the fraction of worms surviving the interval between treatments and $\Delta_{j}$ is the average number of adult worms newly acquired per host in $\left(t_{j}, t_{j+1}\right)$ that survive to the end of this period. The parameter, $\Delta_{j}$, is defined by the model for mean worm burden of the previous section.

We use the model of the previous section for the dynamics of mean microfilarial load per mg skin in the host population to describe the expected microfilarial load per mg skin within an individual host, except that we replace the initial mean worm burden $\left(\tilde{w}_{0}\right)$ with the initial worm burden of the individual $\left(w_{0}\right)$. To obtain the expected microfilarial load of a skin snip sample, we multiply by the combined weight of the two skin-snips that were taken from each individual.

Specifically, for the skin-snip sample taken immediately prior to treatment $j$ at the end of the interval $\left(t_{j-1}, t_{j}\right)$ the expected microfilarial load for an individual with initial worm burden $w_{0}$ is $m_{j-1}^{+}\left(t_{j}\right) d_{j}$, where $d_{j}$ is the combined weight of the two skin snips and

$m_{j-1}^{+}\left(t_{j}\right)=\left\{\begin{array}{ll}\phi^{(0)} w_{0} /\left(2 \mu_{m}\right) & j=1 \\ \frac{1-e^{-\mu_{m}\left(t_{j}-t_{j-1}\right)}}{2 \mu_{m}} \sum_{n=0}^{j-1} \phi^{(n)} w_{j-1}^{(n)} & j>1\end{array}\right.$.

\section{Observed microfilarial loads}

$\mathrm{Up}$ to this point the model has been deterministic, i.e. conditional upon an initial worm burden and set of parameters it provides the expected microfilarial load for the skin snip sample. To model observed microfilarial load we introduce a stochastic element which arises from the distribution of microfilariae within a host's skin. For randomly distributed microfilariae, a Poisson distribution would be appropriate. However, it is likely that there is 'clumping' of microfilariae in the skin (Kershaw, Duke and Budden, 1954), leading to an excess of zeros in comparison with the Poisson distribution (Grenfell et al. 1990; Pion et al. 2006). We have chosen to model the observed distribution of microfilariae with a zero-inflated Poisson distribution. Mechanistically this distribution may be thought of in terms of selecting a skin-snip sample from a 'clumped' versus an 'unclumped' area (note that this interpretation is slightly artificial since we have for simplicity combined data from two skin snips). Within 'clumped' areas we assume that the distribution of an individual's microfilarial load at time $t_{j}$ is Poisson with probability mass function $f(x)$ and mean $d_{j} m_{j-1}^{+}\left(t_{j}\right)$. If the probability of selecting from an 'unclumped' area is $\pi$, then the probability mass function for observed microfilarial load, $x$, is $\pi+(1-\pi) f(x)$ when $x=0$ and $(1-\pi) f(x)$ for $x>0$.

\section{Model fitting}

Estimates were obtained for a restricted set of parameters (Table 1) using a hierarchical Bayesian approach. These include, for each village, the mean worm burden per host prior to treatment and the per female worm rate of microfilarial production per mg of skin after $n$ treatments. For some parameters, estimation was unnecessary since their values were known, i.e. skin-snip weights - recorded for each individual, and fraction of hosts treated at each round and village - given in Table 1 of Collins et al. (1992). It was necessary to fix other parameters to improve the identifiability of the model, i.e. to estimate the parameters from the data with acceptable precision (Huang, 2005). Fixed parameters were: the death rates of microfilariae and of adult worms, and pre-treatment rate of microfilarial production by female worms-taken from Table 2 of Basáñez and Boussinesq (1999). The parameters for pretreatment worm distribution and the zero-inflation parameter, $\pi$, of the microfilarial distribution were also estimated for each village.

The hierarchical Bayesian approach involved estimating parameters associated with the distribution of initial worm burdens. Specifically, we assumed that the initial worm burdens, $\mathbf{w}_{\mathbf{0}}$, are independent draws from a negative binomial distribution with parameters $\tilde{w}_{0}$ and $k$ representing respectively the mean and degree of overdispersion.

To ensure positive estimates of $\phi^{(n)}, \tilde{w}_{0}$, and $k$ these parameters were log-transformed, while $\pi$ was logittransformed since it is constrained to be between 0 and 1 . The set of transformed parameters, $\lambda$, is

$$
\begin{array}{ll}
\lambda_{n}=\log \left(\phi^{(n)}\right)(n=1, \ldots 4) & \lambda_{5}=\operatorname{logit}(\pi) \\
\lambda_{6}=\log \left(\tilde{w}_{0}\right) & \lambda_{7}=\log (k) .
\end{array}
$$

It is convenient to form two groups of parameters $\lambda_{\mathbf{m}}=\left(\lambda_{1}, \ldots, \lambda_{5}\right)$ and $\lambda_{\mathbf{w}}=\left(\lambda_{6}, \lambda_{7}\right)$; the former are parameters associated with the distribution of microfilarial burden, whereas the latter are parameters that specify the distribution of initial worm burden in individuals who took all five treatments.

Given the initial worm burdens, $\mathbf{w}_{\mathbf{0}}$, and parameters $\lambda_{\mathbf{m}}$, the likelihood of the observed microfilarial loads, $\mathbf{x}$, is defined by the zero-inflated Poisson distribution described in the previous section.

The posterior distribution for the transformed parameters can be expressed in terms of this likelihood $\mathrm{p}\left(\mathbf{x} \mid \lambda_{\mathbf{m}}, \mathbf{w}_{\mathbf{0}}\right)$, the distribution of initial worm burdens $\mathrm{p}\left(\mathbf{w}_{\mathbf{0}} \mid \lambda_{\mathbf{w}}\right)$, and the prior $\mathrm{p}(\lambda)$. Up to a constant of proportionality the posterior distribution is

$\mathrm{p}\left(\lambda, \mathbf{w}_{\mathbf{0}} \mid \mathbf{x}\right) \propto \mathrm{p}\left(\mathbf{x} \mid \lambda_{\mathbf{m}}, \mathbf{w}_{\mathbf{0}}\right) \mathrm{p}\left(\mathbf{w}_{\mathbf{0}} \mid \lambda_{\mathbf{w}}\right) \mathrm{p}(\lambda)$. 
Table 1. Parameter/variable definitions

\begin{tabular}{|c|c|c|}
\hline $\begin{array}{l}\text { Parameter/ } \\
\text { variable }\end{array}$ & Definition & Value \\
\hline$w_{j}^{(n)}(t)$ & $\begin{array}{l}\text { The number of worms within a host that have } \\
\text { survived } n \text { treatments at time } t \text { in }\left(t_{j}, t_{j+1}\right)\end{array}$ & \\
\hline$\tilde{w}_{j}^{(n)}(t)$ & $\begin{array}{l}\text { The average number of worms within the } \\
\text { population of hosts to have received } n \text { treatments }\end{array}$ & \\
\hline$\tilde{m}_{j}^{+}(t)$ & $\begin{array}{l}\text { Average microfilarial load per mg of skin among } \\
\text { treated hosts }\end{array}$ & \\
\hline$\tilde{m}_{j}^{-}(t)$ & $\begin{array}{l}\text { Average microfilarial load per mg of skin among } \\
\text { untreated hosts }\end{array}$ & \\
\hline$\tilde{m}_{j}(t)$ & $\begin{array}{l}\text { Average microfilarial load per mg skin in the host } \\
\text { population }\end{array}$ & \\
\hline$d_{j}$ & $\begin{array}{l}\text { Weight of skin snip sample taken immediately prior } \\
\text { to the } j \text { th treatment }\end{array}$ & \\
\hline$f_{j}$ & Fraction of census population treated at treatment $j$ & See Table $1 \dagger$ \\
\hline$q$ & $\begin{array}{l}\text { Product of the rate of transmission via simuliid } \\
\text { vector and the probability that the L } 3 \text { larvae } \\
\text { survive maturation within the host }\end{array}$ & $2 \mu_{m} \mu_{w} / \phi^{(0)}$ \\
\hline$k$ & $\begin{array}{l}\text { Overdispersion of the initial distribution of worm } \\
\text { burden }\end{array}$ & estimated \\
\hline$\pi$ & $\begin{array}{l}\text { Zero-inflation parameter of microfilarial } \\
\text { distribution }\end{array}$ & estimated \\
\hline$\tilde{w}_{0}$ & Mean worm burden prior to treatment & estimated \\
\hline$\phi^{(n)}$ & $\begin{array}{l}\text { Per female worm rate of microfilarial production } \\
\text { per mg of skin after } n \text { treatments }\end{array}$ & estimated \\
\hline$\phi^{(0)}$ & $\begin{array}{l}\text { Per female worm rate of microfilarial production } \\
\text { per mg of skin in the absence of treatment }\end{array}$ & $0 \cdot 7 *$ \\
\hline$\mu_{m}$ & Per capita death rate of microfilariae & $0 \cdot 8^{*}$ \\
\hline$\mu_{w}$ & Per capita death rate worms & $0 \cdot 1 *$ \\
\hline$p$ & $\begin{array}{l}\text { Proportion of established worms that survive the } \\
\text { interval between treatments }\end{array}$ & $e^{-\mu_{w}\left(t_{j+1}-t_{j}\right)}$ \\
\hline
\end{tabular}

All rates are per year. Data source * Basáñez and Boussinesq (1999) † Collins et al. (1992).

All variables are indexed by treatment number $j$. Variables $w_{j}^{(n)}(t), \tilde{w}_{j}^{(n)}(t), \tilde{m}_{j}^{+}(t)$, $\tilde{m}_{j}^{-}(t), \tilde{m}_{j}(t)$ are functions of time $t$.

We used a prior for $\mathrm{p}(\lambda)$ in which the transformed parameters were assigned independent normal priors each with standard deviation of 100 ; for parameters $\lambda_{1}-\lambda_{4}$ the mean of the prior was $\log (0 \cdot 7)$, and for parameters $\lambda_{5}, \lambda_{6}$ and $\lambda_{7}$ the means were respectively $\operatorname{logit}(0 \cdot 5), \log (50)$ and $\log (1)$. To sample from the posterior distribution we used the $\mathrm{McMC}$ algorithm described below. The model parameters, definitions, and sources are listed in Table 1.

\section{McMC algorithm}

Draws were obtained from the posterior distribution using the Gibbs sampler. The Gibbs algorithm works by iteratively sampling from the distribution of each parameter, conditional on the data and on the remaining parameters being fixed at their current values (Gelman et al. 2004). Specifically, samples were drawn from $\mathrm{p}\left(\mathbf{w}_{0} \mid \lambda, \mathbf{x}\right)$ and then from $\mathrm{p}\left(\lambda_{\mathrm{i}} \mid \lambda_{-\mathrm{i}}\right.$, $\left.\mathbf{w}_{\mathbf{0}}, \mathbf{x}\right),(i=1, \ldots, 7)$ where $\lambda_{\mathrm{i}}$ is the $i$ th parameter and $\lambda_{-\mathrm{i}}$ is the parameter vector, $\lambda$, excluding the $i$ th parameter.

The conditional distributions $\mathrm{p}\left(\mathbf{w}_{\mathbf{0}} \mid \lambda, \mathbf{x}\right)$ and $\mathrm{p}\left(\lambda_{\mathrm{i}} \mid \lambda_{-i}, \quad \mathbf{w}_{0}, \mathbf{x}\right)$ are known up to a constant of proportionality from eqn 10 . Samples were obtained from $\mathrm{p}\left(\mathbf{w}_{\mathbf{0}} \mid \lambda, \mathbf{x}\right)$ using the Adaptive Rejection Metropolis Sampling (ARMS) algorithm (Gilks, Best and Tan, 1995), and from $\mathrm{p}\left(\lambda_{\mathrm{i}} \mid \lambda_{-\mathrm{i}}, \mathbf{w}_{\mathbf{0}}, \mathbf{x}\right)$ using the Metropolis algorithm (Metropolis et al. 1953).

The McMC algorithm was programmed using the R programming language (http://www.r-project. org), and the ARMS algorithm was implemented using the arms function from the HI library. The algorithm used in arms gives real-valued samples, not integers. To sample from the conditional distribution of initial worm burden $\mathrm{p}\left(\mathbf{w}_{\mathbf{0}} \mid \lambda, \mathbf{x}\right)$, the output of the arms algorithm was rounded and used as the proposal distribution in a final Metropolis step.

The model was fitted separately to data from each of the three villages. Three sets of simulations (chains) were produced for each village. We used microfilarial load at treatment 1 multiplied by $2 \mu_{m} /$ $\left(\phi^{(0)} d_{1}\right)$ (see eqn 9) as starting values for the initial worm burden $\left(w_{0}\right)$ in all three chains. For the remaining parameters different starting values were chosen for each of the three chains. The starting values were 'overdispersed', i.e. they were selected to be outside the simulated posterior distributions. 
Table 2. Parameter estimates with $95 \%$ Bayesian credible intervals

\begin{tabular}{lccc}
\hline \hline Parameter & Los Tarrales & Santa Emilia & \multicolumn{1}{l}{ Los Andes } \\
\hline$\phi_{1}$ & $0 \cdot 33(0 \cdot 31-0 \cdot 36)$ & $0 \cdot 19(0 \cdot 17-0 \cdot 21)$ & $0 \cdot 24(0 \cdot 22-0 \cdot 26)$ \\
$\phi_{2}$ & $0 \cdot 36(0 \cdot 34-0 \cdot 38)$ & $0 \cdot 20(0 \cdot 18-0 \cdot 23)$ & $0 \cdot 24(0 \cdot 21-0 \cdot 27)$ \\
$\phi_{3}$ & $0 \cdot 08(0 \cdot 07-0 \cdot 09)$ & $0 \cdot 01(0 \cdot 00-0 \cdot 03)$ & $0 \cdot 07(0 \cdot 05-0 \cdot 08)$ \\
$\phi_{4}$ & $0 \cdot 25(0 \cdot 24-0 \cdot 27)$ & $0 \cdot 24(0 \cdot 21-0 \cdot 28)$ & $0 \cdot 21(0 \cdot 19-0 \cdot 24)$ \\
$\tilde{w}_{0}$ & $44(36-54)$ & $46(36-64)$ & $51(38-69)$ \\
$k$ & $0 \cdot 38(0 \cdot 32-0 \cdot 44)$ & $0 \cdot 38(0 \cdot 30-0 \cdot 48)$ & $0 \cdot 29(0 \cdot 23-0 \cdot 38)$ \\
$\pi$ & $0 \cdot 32(0 \cdot 29-0 \cdot 35)$ & $0 \cdot 39(0 \cdot 33-0 \cdot 44)$ & $0 \cdot 42(0 \cdot 37-0 \cdot 46)$ \\
\hline \hline
\end{tabular}

Each chain was run for 2000 iterations. The three sequences appeared to have converged after 1000 iterations. We treated the first half of each chain as 'burn-in' and used the second half of each chain to provide a sample of 3000 on which inference was based.

\section{Sensitivity analysis}

Sensitivity or uncertainty analysis permits exploration of how model outcomes are affected by changes in structural assumptions (biological hypotheses and their mathematical formulation) or changes in parameter values (Haefner, 1996). To explore the sensitivity of the results to changes in the parameter value chosen for $\phi^{(0)}$ (the pre-treatment rate of microfilarial production) we used the endpoints of the range given in Table 2 of Basáñez and Boussinesq (1999) (i.e. $\phi^{(0)}=0.54$ and $0.79 \mathrm{yr}^{-1}$ female $^{-1} \mathrm{mg}$ of $\operatorname{skin}^{-1}$ ). In addition the potential impact of nodulectomy on the results was assessed, albeit approximately, by performing a sensitivity analysis in which the death rate of worms post treatment was increased to $\mu_{w}=0 \cdot 2 \mathrm{yr}^{-1}$ (compared to a pre-treatment value of $0 \cdot 1 \mathrm{yr}^{-1}$ ).

\section{RESULTS}

Table 2 presents the estimates for the average rates of microfilarial production corresponding to the 6-month period following each treatment round; the estimated mean adult worm burden at baseline in those individuals that took all five treatments; the overdispersion parameter for the initial distribution of adult worms among hosts, and the zero-inflation parameter for the distribution of microfilarial loads. Each parameter estimate is presented with its associated $95 \%$ Bayesian credible interval. Excluding the values for treatment 3 , the values for the average rate of microfilarial production 6 months after the previous treatment varied between $0 \cdot 19$ (Santa Emilia, treatment 1) and $0 \cdot 36$ (Los Tarrales, treatment 2 ), averaging $0 \cdot 25$ microfilariae per $\mathrm{mg}$ of skin, per female worm per year. This represents a reduction of $64 \%$ from the initial value of 0.7 presented by Basáñez and Boussinesq (1999). The values corresponding to the third treatment are very low across the three villages but do not indicate a systematic downward trend. The values of the mean initial worm burden per host and overdispersion parameter among hosts are similar for all three villages averaging to 47 (mean) and $0 \cdot 35$ (overdispersion).

Estimates of the relative change in microfilarial production are robust to changes in the value of $\phi^{(0)}$. For both $\phi^{(0)}=0.54$ and $\phi^{(0)}=0.79$ microfilariae per female worm per year and per mg of skin, the estimated rate of microfilarial production is $37 \%$ of the pre-treatment value (compared to $36 \%$ in the original simulation where $\left.\phi^{(0)}=0 \cdot 7\right)$. By contrast, increasing the death rate of adult worms post treatment appeared to increase estimates of microfilarial production. Setting $\mu_{w}=0.2$ after treatment (pretreatment $\mu_{w}=0.1$ per year) the estimate of production increased to $42 \%$ of the pre-treatment value (i.e. an overall reduction of $58 \%$ compared with $64 \%$ for the nominal value). This agrees with intuition, which suggests that for the same observed microfilaridermia the per worm microfilarial production must be higher if there are fewer adult worms as a result of nodulectomy (life-expectancy reduced from 10 years to 5 years).

\section{DISCUSSION}

Our results (Table 2) show a substantial reduction in the rate of microfilarial production during the six months after the first treatment. On average, the per capita rate of microfilarial production (scaled per $\mathrm{mg}$ of skin) was reduced to $\sim 36 \%$ of its pre-treatment (nominal) value of 0.7 microfilariae per female per year. (This pre-treatment value had been estimated by Basáñez and Boussinesq (1999) by combining rates of microfilarial release by adult worms maintained in vitro (Engelbrecht and Schulz-Key, 1984) with in vivo estimates presented by Duke (1993).) However, we did not find, unlike Plaisier et al. (1995), that subsequent treatments reduced cumulatively the rate of microfilarial production by O. volvulus.

Estimates of microfilarial production were similarly reduced for the six-month periods following treatments 2 and 4, but were significantly lower for the six months after treatment 3. This pattern was consistent across the 3 communities. A reduction in microfilarial loads at treatment 3 followed by an increase at treatment 4 had been observed by Collins 
et al. (1992) The low rate of microfilarial production following treatment 3 is difficult to explain biologically since it does not appear to be part of a systematic downward trend. A possibility is that this result may simply reflect a change in the way the sample was collected or analysed at this treatment time. Other explanations include the possible effects of nodulectomy on worm burden, and the observation that during the trial transmission of L3 by S. ochraceum s.l. was substantially reduced (Cupp et al. 1992). The latter effect, however, is likely to be small since the reduction in transmission due to treatment is incorporated into the model; furthermore even with complete interruption of transmission, the adult worm population would only decline by approximately $5 \%$ per six months (assuming an average lifeexpectancy of 10 years for adult worms as in Basáñez and Boussinesq (1999) and Plaisier et al. (1991)). A reduced worm life-expectancy, due to nodulectomy may be responsible for a larger reduction in the size of the worm population between treatment 2 and treatment 3. Furthermore, this reduction in the size of the worm population may cause a decline in mating probability and insemination rates which will in turn further reduce observed microfilarial loads. However, this does not explain the recovery of microfilarial production at treatment 4 , unless this is itself an anomalous result.

The estimated average reduction in microfilarial production 6 months after ivermectin treatment is consistent with the results of other studies on the evolution of microfilarial load following treatment. In a study of 5 yearly treatments in Asubende, Ghana, Plaisier et al. (1995) fitted a model to the data in which female worms ceased producing microfilariae immediately after treatment, and then gradually recovered microfilarial production with time postivermectin. They estimated that microfilarial production had reached a value of approximately $68 \%$ of the pre-treatment level 10 months after treatment. This corresponds to an average rate of microfilarial production during the first six months of $20 \%$ of the pre-treatment value assuming microfilarial production increases linearly during this period.

Duke et al. (1991) found in a longitudinal study of reproductive activity by $O$. volvulus after ivermectin treatment in Guatemala, that 6 months posttreatment only 17 out of 53 live females (32\%, $95 \%$ $\mathrm{CI}=0 \cdot 20,0.45)$ were releasing live microfilariae. This finding is less easy to compare to our result although the two may be consistent if ivermectin eliminates microfilarial production in a proportion of worms and has little or no effect in the remaining worms, as proposed by Plaisier et al. (1995).

Further comparisons are possible by using the model to predict microfilarial loads post treatment. For a static worm population whose rate of microfilarial production remains at $36 \%$ of the pretreatment level during the period 6-12 months following treatment, the expected microfilarial load at 12 months is predicted to be $20 \%$ of the pretreatment load (from eqns 3 and 4). This figure is in agreement with the findings of a recent meta-analysis and mathematical model by Basáñez et al. (2008), in which average microfilarial loads are $\sim 20 \%$ of their pre-treatment levels one year after single-dose ivermectin.

Mean baseline microfilarial load was similar across the villages (Los Tarrales: 27.0 microfilariae per $\mathrm{mg}$; Santa Emilia: 23.5 microfilariae per $\mathrm{mg}$, and Los Andes: 18.6 microfilariae per $\mathrm{mg}$ ). This is reflected in similar estimates of worm burden among the three villages, averaging 47 worms per person. Basáñez et al. (2002) analyzed data on microfilarial loads collected by Brandling-Bennett et al. (1981), approximately 10 years earlier than the data used in this study. They found a higher microfilarial load per $\mathrm{mg}$ of skin in Santa Emilia $(64 \cdot 3)$, but similar loads in Los Tarrales (18.2) and Los Andes (28.9). This lends support to the hypothesis of endemic equilibrium prior to treatment, particularly in the latter two villages. Although it appears less plausible that Santa Emilia may have been at endemic equilibrium preivermectin, this may not significantly affect our results. This is because the analysis undertaken requires only that mean worm burden should be approximately constant for the 12 months preceding the introduction of chemotherapy (the assumed length of the maturation period from L3 to adult worm used here). The assumption may be reasonable even if the system is not at endemic equilibrium, since it is unlikely that adult worm numbers would have changed considerably within a 12-month period. Also, mean microfilaridermia at the two timepoints may not be strictly equivalent, as the data published in 1981 and analysed by Basáñez et al. (2002) referred to all the population examined whereas the data published in 1992 and analysed in this paper refers to the subset of the population who adhered to all 5 treatment rounds.

In this study we lacked data on the numbers of worms removed by nodulectomy from each participant at each treatment for the reasons mentioned above; we can therefore not exclude the possibility that in addition to ivermectin effects, some of the measured reduction in microfilarial load may be due to nodulectomy (via increased worm mortality). However, the sensitivity analysis shows that even a doubling in the rate of worm mortality post ivermectin has only a modest effect on the estimate of worm fertility. It is also reassuring that the estimated effects are in agreement with those measured in other studies not confounded by excision of onchocercomata (Plaisier et al. 1995; Basáñez et al. 2008). In addition to eliminating a potential source of bias, nodulectomy data would also provide a means of validating the estimates of mean worm burden using the relationship between numbers of adult worms 
and excisable (palpable) onchocercomata developed by Duerr et al. (2001). According to their model we would expect an average of approximately 10 palpable nodules per person.

\section{Concluding remarks and future directions}

One of the strengths of these data is that they may be used to estimate the effect of successive treatments on microfilarial production by the adult female worms. This is important in order to further our understanding of the long-term impact of ivermectinbased onchocerciasis control programmes. Our estimates suggest that the production of microfilariae is not cumulatively reduced by successive ivermectin treatments over the 30 -month study period. However, this does not rule out the possibility that longerterm, multiple ivermectin treatments may lead to a decline in the rate of microfilarial production by the adult worms, or to other changes in the reproductive biology of the parasite such as female-biased (intranodular) sex ratios, decreased mating probabilities and insemination rates, and increased macrofilaricidal effects (equivalent to irreversible sterilisation of adult worms) (Duke et al.1991; Gardon et al. 2002; Cupp et al. 2004; Cupp and Cupp, 2005).

The only other model that has been fitted to timedependent microfilarial data from multiple regular treatments is that of Plaisier et al. (1995). Although their modelling approach is different from ours, and the frequency of treatment in Asubende, Ghana (Alley et al. 1994) was annual rather than 6-monthly as in Guatemala, the results of both studies are compatible and also comparable to those obtained by Basáñez et al. (2008), who modelled the effect of a single ivermectin dose. However, Plaisier et al. (1995) found that a progressive reduction of microfilarial production with each dose would be the scenario most consistent with the data. It would be very interesting to reanalyse such data with the modelling approach presented here, as projections of onchocerciasis elimination assume operation of such cumulative effects (Winnen et al. 2002).

In order to extend our model beyond the 30 -month study period with the aim to investigate the probability of onchocerciasis elimination, we would also have to acknowledge that $q$ (the per microfilaria rate of success in becoming an established worm) is likely to be strongly nonlinear, as density dependence has been identified to operate upon microfilarial uptake and survival, parasite establishment, vector and human survival, and the biting rate per fly on humans (Basáñez et al. 1995, 1996, 2002, 2007; Duerr et al. 2003 ; Little et al. 2004).

In conclusion, our ability to project the long-term impact of ivermectin on transmission and the possibility of infection elimination with microfilaricidal chemotherapy alone depends on improving our understanding of the effects of repeated treatment on the population biology of $O$. volvulus. Modelling analyses of further time-series of microfilarial loads and/or of the reproductive and viability status of adult parasites obtained from repeatedly treated patients (such as the data presented by Gardon et al. (2002)) will be required to clarify the effect of longterm ivermectin treatment, and any changes in treatment efficacy (Osei-Atweneboana et al. 2007) that may result from chemotherapy-induced selection pressure acting upon the parasites genome (Bourguinat et al. 2007, 2008).

The data analysed in this paper were collected during a study funded by the UNDP/World Bank/WHO Special Program for Research and Training in Tropical Disease (project ID870340) conducted in Guatemala between 1988 and 1990. CB would like to thank the Department of Statistical Science at University College London for financial support provided during the preparation of this paper. M-GB acknowledges the Medical Research Council, UK for a Career Establishment Grant.

\section{REFERENCES}

Alley, E. S., Plaisier, A. P., Boatin, B. A., Dadzie, K. Y., Remme, J., Zerbo, G. and Samba, E. M. (1994). The impact of five years of annual ivermectin treatment on skin microfilarial loads in the onchocerciasis focus of Asubende, Ghana. Transactions of the Royal Society of Tropical Medicine and Hygiene 88, 581-584.

Basáñez, M.-G. and Boussinesq, M. (1999). Population biology of human onchocerciasis. Philosophical Transactions of the Royal Society of London B 354, 809-826.

Basáñez, M.-G., Collins, R. C., Porter, C. H., Little, M. P. and Brandling-Bennett, D. (2002).

Transmission intensity and the patterns of Onchocerca volvulus infection in human communities. American Fournal of Tropical Medicine and Hygiene 67, 669-679.

Basáñez, M.-G., Pion, S. D. S., Boakes, E., Filipe, J. A. N., Churcher, T. S. and Boussinesq, M. (2008). Effect of single-dose ivermectin on Onchocerca volvulus: a systematic review and meta-analysis. Lancet Infectious Diseases 8, 310-322.

Basáñez, M.-G., Pion, S. D. S., Churcher, T. S., Breitling, L. P., Little, M. P. and Boussinesq, $M$. (2006). River blindness: a success story under threat? PLoS Medicine 3, e371.

Basáñez, M.-G., Razali, K., Renz, A. and Kelly, D. (2007). Density-dependent host choice by disease vectors: epidemiological implications of the ideal free distribution. Transactions of the Royal Society of Tropical Medicine and Hygiene 101, 256-269.

Basáñez, M.-G., Remme, J. H., Alley, E. S., Bain, O., Shelley, A. J., Medley, G. F. and Anderson, R. M. (1995). Density-dependent processes in the transmission of human onchocerciasis: relationship between the numbers of microfilariae ingested and successful larval development in the simuliid vector. Parasitology 110, 409-427.

Basáñez, M.-G., Townson, H., Williams, J. R., Frontado, H., Villamizar, N. J. and Anderson, R. M. (1996). Density-dependent processes in the transmission of human onchocerciasis : relationship 
between microfilarial intake and mortality of the simuliid vector. Parasitology 113, 331-355.

Bourguinat, C., Ardelli, B. F., Pion, S. D. S., Kamgno, J., Gardon, J., Duke, B. O. L., Boussinesq, M. and Prichard, R. K. (2008). P-glycoprotein-like protein, a possible genetic marker for ivermectin resistance selection in Onchocerca volvulus. Molecular and Biochemical Parasitology 158, 101-111.

Bourguinat, C., Pion, S. D. S., Kamgno, J., Gardon, J., Duke, B. O. L., Boussinesq, M. and Prichard, R. K. (2007). Genetic selection of low fertile Onchocerca volvulus by ivermectin treatment. PLoS Neglected Tropical Diseases 1, e72.

Brandling-Bennett, A. D., Anderson, J., Fuglsang, H. and Collins, R. (1981). Onchocerciasis in Guatemala. Epidemiology in fincas with various in tensities of infection. American Fournal of Tropical Medicine and Hygiene 30, 970-981.

Collins, R. C. (1979). Onchocerciasis transmission potentials of four species of Guatemalan simuliidae. American Fournal of Tropical Medicine and Hygiene 28, $72-75$.

Collins, R. C., Campbell, C. C., Wilton, D. P. and Newton, L. (1977). Quantitative aspects of the infection of Simulium ochraceum by Onchocerca volvulus. Tropenmedizin und Parasitologie 28, 235-243.

Collins, R. C., Gonzales-Peralta, C., Castro, J., Zea-Flores, G., Cupp, M. S., Richards, F. O. J. and Cupp, E. W. (1992). Ivermectin: reduction in prevalence and infection intensity of Onchocerca volvulus following biannual treatments in five Guatemalan communities. American Fournal of Tropical Medicine and Hygiene 47, 156-169.

Cupp, E. W. and Cupp, M. S. (2005). Impact of ivermectin community level treatments on elimination of adult Onchocerca volvulus when individuals receive multiple treatments per year. American Fournal of Tropical Medicine and Hygiene 73, 1159-1161.

Cupp, E. W., Duke, B. O. L., Mackenzie, C. D., Guzmán, J. R., Vieira, J. C., Mendez-Galván, J., Castro, J., Richards, F., Sauerbrey, M., Dominguez, A., Eversole, R. R. and Cupp, M. S. (2004). The effects of long-term community level treatment with ivermectin (Mectizan) on adult Onchocerca volvulus in Latin America. American Fournal of Tropical Medicine and Hygiene 71, 602-607.

Cupp, E. W., Ochoa, J. O., Collins, R. C., Cupp, M. S., Gonzales-Peralta, C., Castro, J. and Zea-Flores, G. (1992). The effects of repetitive community-wide ivermectin treatment on transmission of Onchocerca volvulus in Guatemala. American Fournal of Tropical Medicine and Hygiene 47, 170-180.

Diallo, S., Aziz, M. A., Larivière, M., Diallo, J. S., Diop-Mar, I., N'Dir, O., Badiane, S., Py, D., Schulz-Key, H. and Gaxotte, P. (1986). A doubleblind comparison of the efficacy and safety of ivermectin and diethylcarbamazine in a placebo controlled study of Senegalese patients with onchocerciasis. Transactions of the Royal Society of Tropical Medicine and Hygiene 80, 927-934.

Duerr, H. P., Dietz, K., Buttner, D. W. and Schulz-Key, H. (2001). A stochastic model for the aggregation of Onchocerca volvulus in nodules. Parasitology 123, 193-201.
Duerr, H. P., Dietz, K. and Eichner, M. (2005). Determinants of the eradicability of filarial infections: a conceptual approach. Trends in Parasitology 21, 88-96.

Duerr, H. P., Dietz, K., Schulz-Key, H., Büttner, D. W. and Eichner, M. (2003). Density-dependent parasite establishment suggests infection-associated immunosuppression as an important mechanism for parasite density regulation in onchocerciasis. Transactions of the Royal Society of Tropical Medicine and Hygiene 97, 242-250.

Duke, B. O. L. (1980). Observations on Onchocerca volvulus in experimentally infected chimpanzees. Tropenmedizin und Parasitologie 31, 41-54.

Duke, B. O. L. (1993). The population dynamics of Onchocerca volvulus in the human host. Tropical Medicine and Parasitology 44, 61-68.

Duke, B. O. L., Zea-Flores, G. and Muñoz, B. (1991). The embryogenesis of Onchocerca volvulus over the first year after a single dose of ivermectin. Tropical Medicine and Parasitology 42, 175-180.

Engelbrecht, F. and Schulz-Key, H. (1984). Observations on adult Onchocerca volvulus maintained in vitro. Transactions of the Royal Society of Tropical Medicine and Hygiene 78, 212-215.

Gardon, J., Boussinesq, M., Kamgno, J., GardonWendel, N., Demanga-Ngangue and Duke, B. O. L. (2002). Effects of standard and high doses of ivermectin on adult worms of Onchocerca volvulus: a randomised controlled trial. Lancet 360, 203-210.

Gelman, A., Carlin, J. B., Stern, H. S. and Rubin, D. B. (2004). Bayesian Data Analysis, 2nd Edn. Chapman and Hall/CRC, Boca Raton.

Gilks, W. R., Best, N. G. and Tan, K. K. C. (1995). Adaptive rejection Metropolis sampling within Gibbs sampling. Applied Statistics 44, 455-472.

Grenfell, B. T., Das, P. K., Rajagopalan, P. K. and Bundy, D. A. P. (1990). Frequency distribution of lymphatic filariasis microfilariae in human populations: population processes and statistical estimation. Parasitology 101, 417-427.

Haefner, J. W. (1996). Modeling Biological Systems. Principles and Applications. Chapman and Hall, New York.

Huang, G.-H. (2005). Model Identifiability. In Encyclopedia of Statistics in Behavioral Science Volume 3 (ed. Everitt, B. S. and Howell, D. C.), pp. 1249-1251. John Wiley \& Sons, Chichester.

Kershaw, W. E., Duke, B. O. L. and Budden, F. H. (1954). Distribution of microfilariae of $O$. volvulus in the skin; its relation to the skin changes and to eye lesions and blindness. British Medical Fournal 2, 724-729.

Kläger, S., Whitworth, J. A., Post, R. J., Chavasse, D. C. and Downham, M. D. (1993). How long do the effects of ivermectin on adult Onchocerca volvulus persist? Tropical Medicine and Parasitology 44, 305-310.

Little, M. P., Breitling, L. P., Basáñez, M.-G., Alley, E. S. and Boatin, B. A. (2004). Association between microfilarial load and excess mortality in onchocerciasis : an epidemiological study. Lancet 363, 1514-1521.

Meredith, S. E. O. and Dull, H. B. (1998).

Onchocerciasis: the first decade of Mectizantrade mark treatment. Parasitology Today 14, 472-474.

Metropolis, N., Rosenbluth, A. W., Rosenbluth, M. N., Teller, A. H. and Teller, E. (1953). Equations 
of state calculations by fast computing machines. Fournal of Chemical Physics 21, 1087-1092.

Molyneux, D. H. and Davies, J. B. (1997).

Onchocerciasis control: moving towards the millennium. Parasitology Today 13, 418-425.

Osei-Atweneboana, M. Y., Eng, J. K., Boakye, D. A., Gyapong, J. O. and Prichard, R. K. (2007). Prevalence and intensity of Onchocerca volvulus infection and efficacy of ivermectin in endemic communities in Ghana: a two-phase epidemiological study. Lancet 369, 2021-2029.

Pion, S. D. S., Filipe, J. A. N., Kamgno, J., Gardon, J., Basáñez, M.-G. and Boussinesq, M. (2006).

Microfilarial distribution of Loa loa in the human host: population dynamics and epidemiological implications. Parasitology 133, 101-109.

Plaisier, A. P., Alley, E. S., Boatin, B. A., Van Oortmarssen, G. J., Remme, H., De Vlas, S. J., Bonneux, L. and Habbema, J. D. (1995). Irreversible effects of ivermectin on adult parasites in onchocerciasis patients in the Onchocerciasis Control Programme in West Africa. Fournal of Infectious Diseases 172, 204-210.

Plaisier, A. P., van Oortmarssen, G. J., Remme, J. and Habbema, J. D. (1991). The reproductive lifespan of Onchocerca volvulus in West African savanna. Acta Tropica 48, 271-284.

Richards, F. O., Boatin, B., Sauerbrey, M. and Sékétéli, A. (2001). Control of onchocerciasis today: status and challenges. Trends in Parasitology 17, 558-563.

Schulz-Key, H. and Karam, M. (1986). Periodic reproduction of Onchocerca volvulus. Parasitology Today 2, 284-286.

Winnen, M., Plaisier, A. P., Alley, E. S., Nagelkerke, N. J., van Oortmarssen, G., Boatin, B. A. and Habbema, J. D. (2002). Can ivermectin mass treatments eliminate onchocerciasis in Africa? Bulletin of the World Health Organization 80, 384-391. 